\title{
Performance Measurement-Systeme als Instrumente der unternehmerischen Steuerung
}

\author{
Christian Klatte
}

\section{Notwendigkeit für ein leistungsfähiges Performance Measurement-System}

\subsection{Problemstellung}

Was in den 70er und 80er Jahren von einigen Autoren mit den Stichworten Globalisierung der Märkte, staatliche Deregulierung, zunehmende Dynamik und Komplexität der Umwelten, steigende Innovationsgeschwindigkeit etc. beschrieben wurde, ist heute im Informationszeitalter offensichtlich Realität geworden [1].

Während in den Anbietermärkten des Industriezeitalters der Erfolg einer Unternehmung von der Outputmenge und dem Kostenniveau abhing, entscheiden im Informationszeitalter zusätzliche marktbezogene Anforderungsmerkmale über das Überleben eines Unternehmens. Neben der Wirtschaftlichkeit stellen darüber hinaus die Qualität, die Flexibilität sowie die Innovationskraft einer Unternehmung weitere erfolgskritische Kompetenzen im Wettbewerb um den Kunden dar.

Die Frage die sich in diesem Zusammenhang stellt lautet: Wie können diese schwer fassbaren Kompetenzen systematisch gesteuert und verbessert werden?

Sowohl aufgrund gesetzlicher Bestimmungen als auch aufgrund der fruiheren Erfolgsfaktoren sind traditionelle Steuerungssysteme auf die Darstellung von finanzwirtschaftlichen Kennzahlen und Indikatoren fokussiert. Diese finanziellen Kennzahlen, mit denen die meisten Unternehmen noch heute ihre Geschäfte steuern, tragen den neuen Anforderungen aus folgenden Gründen nur unzureichend Rechnung:

- Sie sind vergangenheitsorientiert.

- Sie geben keine Hinweise auf die Ursachen ihrer Entwicklung.

- Sie sind nur eingeschränkt operationalisierbar.

- Sie verleiten zur kurzfristigen Ergebnisverbesserung zu Lasten langfristiger immaterieller Investitionen.

Darüber hinaus bilden traditionelle Kennzahlensysteme, wie z. B. das ROI-Schema, unabhängig von den unternehmensindividuellen Erfolgsfaktoren stets die gleichen Steuerungsgrößen ab. Eine Auseinandersetzung mit den strategischen Zielen und den relevanten Kunden- und Marktsegmenten unterbleibt. Die Abbildung und Messung von Wertschöpfungsaktivitäten gerät dabei zwangsläufig in den Hintergrund. Innovations-, Wachstums- und Entwicklungspotenziale der Mitarbeiterebene werden zumeist völlig vernachlässigt [2].

Mit der Kritik an den traditionellen Steuerungskonzepten und der steigenden Notwendigkeit nach einem leistungsorientierten Steuerungssystem tauchte seit Mitte der 80er Jahre verstärkt der Begriff Performance Measurement in der englischsprachigen Literatur auf.

\subsection{Begriffsklärung}

Was bedeutet Performance Measurement? R. Gleich versteht unter dem Begriff Performance Measurement den „Aufbau und Einsatz mehrerer quantifizierbarer Maßgrößen verschiedenster Dimensionen (z. B. Kosten, Zeit, Qualität etc.), die zur Beurteilung der Effektivität und Effizienz der Leistung und Leistungspotentiale unterschiedlichster Objekte im Unternehmen (Organisationseinheiten unterschiedlichster Größe, Mitarbeiter, Prozesse) herangezogen werden [3]".

Der Grund, weshalb man geneigt ist, Leistung stets messen zu wollen, ist darin zu sehen, dass ,Leistung' sich nicht unmittelbar zeigt, sondern nur mit Hilfe von quantifizierbaren Größen erfassbar ist. Deshalb liegt dem Kern eines Performance Measurement-Systems das Postulat zu Grunde - ,You can not manage what you can not measure“ [4]. Mit Hilfe der transparenten Darstellung der für den Unternehmenserfolg entscheidenden Faktoren sollen strategieorientierte Ziele herausgestellt und aus diesen konkrete Handlungsempfehlungen abgeleitet werden. Dabei stellt ein Performance Measurement-System kein einmaliges Projekt dar. Ständig uiberarbeitet, trägt es zur kontinuierlichen Verbesserung der Gesamtleistung des Unternehmens bei.

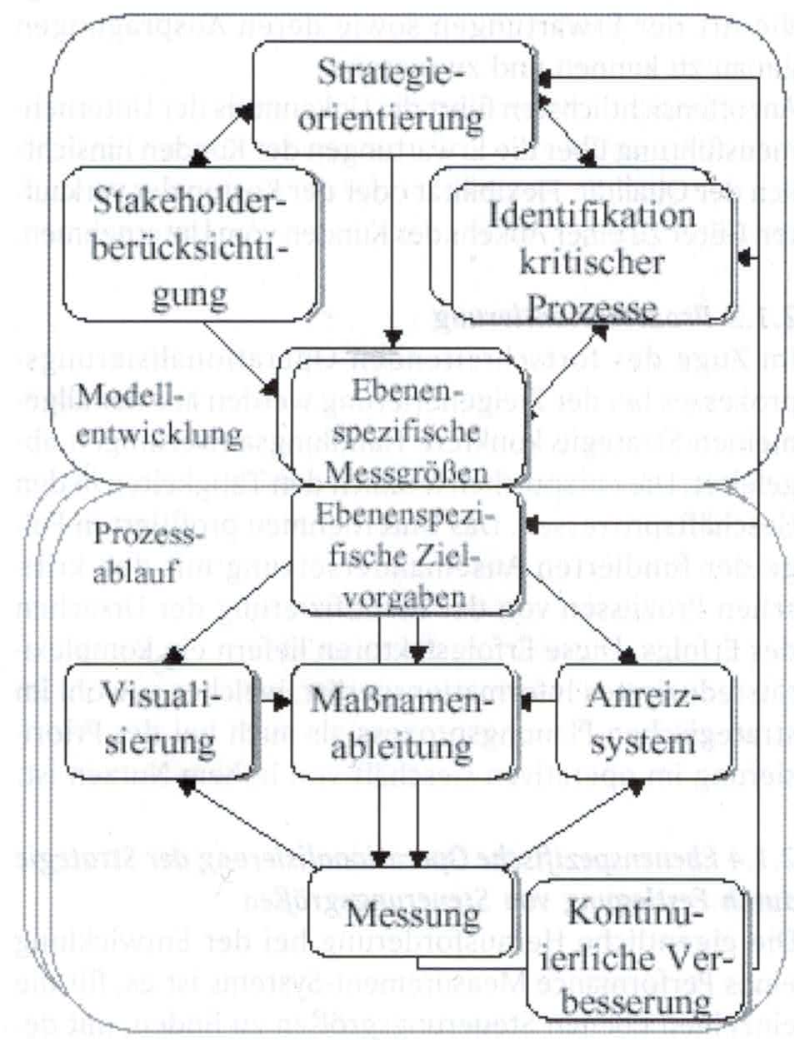

Abb. 1: Charakteristika von Performance Measurement-Systemen 


\section{Charakteristika von Performance Measurement-Systemen}

In der Literatur fallen immer wieder bestimmte Merkmale im Aufbau und Ablauf der Performance MeasurementSysteme auf, die im Folgenden übersichtlich zusammengefasst werden sollen (siehe Abbildung 1).

Diese Bausteine können als Prüfkriterien genutzt werden, um Steuerungssysteme auf ihre Ganzheitlichkeit hin zu beurteilen.

\subsection{Aufbau}

\subsubsection{Strategieorientierung der Steuerungssysteme}

Da die Strategie aufgrund des Unternehmensumfeldes und der unternehmensinternen Stärken und Schwächen einen stark individuellen Charakter trägt, gibt es auch keine universellen Steuerungssysteme. Die Steuerungsgrößen eines Performance Measurement-Systems basieren demnach nicht auf einem starren Kennzahlengerüst, sondern auf den unternehmenseigenen Erfolgsfaktoren. Anstatt einer kurzfristigen Ergebnisverbesserung zielt ein Performance Measurement-System auf die Umsetzung einer langfristigen Strategie.

\subsubsection{Identifizierung der relevanten Stakeholder und deren Ansprüche}

Weil ein Unternehmen neben der Arbeit des Managements auch auf die Leistung von weiteren Anspruchsgruppen angewiesen ist, wie z. B. von Mitarbeitern, Eigentümern und Kunden, müssen deren Ziele ebenfalls bei der Strategiefindung berüicksichtigt werden. Denn nur wenn die an der Wertschöpfung beteiligten Stakeholder ihren Aufwand durch das Unternehmen in ihren Augen befriedigt sehen, werden sie weiterhin im ,Kooperationsverbund' verbleiben. Deshalb ist es notwendig, die Art der Erwartungen sowie deren Ausprägungen genau zu kennen und zu messen.

Am offensichtlichsten führt die Unkenntnis der Unternehmensführung über die Erwartungen der Kunden hinsichtlich der Qualität, Flexibilität oder der Kosten der verkauften Güter zu einer Abkehr des Kunden vom Unternehmen.

\subsubsection{Prozessorientierung}

Im Zuge des fortschreitenden Operationalisierungsprozesses bei der Zielgenerierung werden aus der allgemeinen Strategie konkrete Handlungsanweisungen abgeleitet. Die entsprechen letztlich den Tätigkeiten in den Geschäftsprozessen. Das Unternehmen profitiert in Folge der fundierten Auseinandersetzung mit den kritischen Prozessen von der Identifizierung der Ursachen des Erfolgs. Diese Erfolgsfaktoren liefern ein komplexitätsreduziertes Informationsgerüst, welches sowohl im strategischen Planungsprozess als auch bei der Priorisierung im operativen Geschäft von hohem Nutzen ist.

\subsubsection{Ebenenspezifische Operationalisierung der Strategie durch Festlegung von Steuerungsgrößen}

Die eigentliche Herausforderung bei der Entwicklung eines Performance Measurement-Systems ist es, für die einzelnen Ebenen Steuerungsgrößen zu finden, mit denen der Beitrag eines jeden Einzelnen an der Strategie- erfuillung gemessen werden kann. Abgeleitet werden diese Steuerungsgrößen aus den in der Prozessanalyse identifizierten Erfolgsfaktoren. Ein Kerngedanke eines Performance Measurement-Systems ist der Anspruch auf Ausgewogenheit der Steuerungsgrößen. Aufgrund der Mängel von traditionellen Leistungsmesssystemen wird der ausbalancierte Einsatz von finanziellen und nichtfinanziellen, individuellen und standardisierten sowie prozess- und ergebnisorientierten Kennzahlen gefordert. Die Auswahl der Steuerungsgrößen muss sich auf das Wesentliche und somit auf eine beschränkte Anzahl konzentrieren. Weiterhin sollten sich die Steuerungsgrößen durch eine leichte Verständlichkeit und eine simple Erhebungsmethode auszeichnen.

Sind die Messgrößen ebenenspezifisch unter Berücksichtigung der Stakeholderinteressen und der internen Prozesse sowie der daraus entstehenden Strategie definiert worden, so ist die Grundstruktur eines Performance Measurement-Systems entwickelt.

\subsection{Ablauf}

Obwohl im Detail durchaus unterschiedlich, orientieren sich die meisten Performance Measurement-Systeme bei der Beschreibung des Ablaufprozesses an Demings PlanDo-Check-Act-Kreislauf.

Durch die quantifizierte Vorgabe von operativen Zielen mittels der zuvor definierten Steuerungsgrößen, wissen die Prozessverantwortlichen genau mit welchem Leistungsniveau sie einen Beitrag zur Erreichung der Unternehmensstrategie leisten. Aus den operationalisierten Steuerungsgrößen können konkrete Handlungsanweisungen abgeleitet werden.

Wie aus dem Namen „Performance Measurement“ ersichtlich, ist die Messung der Leistung elementarer Bestandteil des Steuerungsinstruments. Die Messung bildet die Ausgangsbasis fur die Beurteilung der realisierten Leistung. In welchen Abständen ein Zustand gemessen wird, ist abhängig vom Aufwand der Datenerhebung, der relativen Bedeutung der Steuerungsgröße und von der Zeit, die vergeht, bis ein bestimmter Grad der Neuinformation vorliegt. Nachdem der Leistungsstand ermittelt wurde (Ist-Zustand), wird unter Berücksichtigung der Leistungsbedingungen und in Abhängigkeit der Leistungspotenziale die „Performance“ bewertet.

Die Bewertung der Zielerreichung kann durch einen historischen Vergleich, einen internen sowie externen Objektvergleich (Benchmarking) und/oder einem Soll-IstVergleich relativiert werden.

Um die Effektivität des Performance Measurement-Systems weiterhin sicherzustellen, muss regelmäßig durch einen mehrstufigen Feedback-Prozess überprüft werden, ob sich die Ausgangssituation und damit die Basis der kausalen Ableitung der Steuerungsgrößen verändert hat. Neben externen Einfluissen können auch interne Leistungssteigerungen Einfluss auf die Strategie oder auf bestimmte kritische Prozesse haben. Indem beispielsweise die Kosten, die Qualität oder die Entwicklungszeiten eines Produktes verbessert werden, qualifiziert sich das Unternehmen eventuell für einen größeren oder lukrativeren Kundenkreis, dessen Eroberung ebenfalls strategisch geplant sein will. 


\subsection{Instrumente}

Verschiedene Instrumente unterstiitzen die Arbeit innerhalb des Regelkreises.

Mit der Visualisierung von Plandaten und Wirkungszusammenhängen sowie der Kriterien der Leistungsbeurteilung erhöht sich die Transparenz für die Informationsempfänger. Die geschaffene Transparenz steigert die Motivation und hilft den Mitarbeitern, die Auswirkungen ihrer Arbeit auf die Prozesse als auch auf die Strategie besser zu beurteilen. Darüber hinaus werden Widerstände bei der Implementierung eines Performance Measurement-Systems, die aus Ängsten und Unsicherheiten entstehen, minimiert.

In der betrieblichen Praxis setzt sich als Instrument der Visualisierung zunehmend die sogenannte Cockpit-Darstellung durch. Diese liefert individuell relevante Informationen, indem alle persönlichen Steuerungsgrößen und -ziele in meist grafischer Form übersichtlich abgebildet werden. Die Visualisierung schafft durch die Darstellung des Zielerreichungsgrades und weiterer Informationen die Voraussetzungen zur anschaulichen Bewertung des Mitarbeiterverhaltens.

Um das Verhalten der Mitarbeiter strategieorientiert auszurichten und die individuellen Ziele mit denen des Unternehmens zu verknüpfen, müssen den Mitarbeitern Anreize geboten werden. Dabei sind leistungsbezogene Entlohnungsmodelle, wie z. B. die Vergabe von Boni oder Firmenanteilen, nur ein Mittel in einem umfassenden Anreizsystem. Weitere Bestandteile können u. a. die Bewilligung von Freizeit, Belobigungen, Beförderungen oder Weiterbildungen sein. Bevor in einem Unternehmen ein leistungsabhängiges Entlohnungssystem eingeführt werden kann, müssen andere Performance Measurement-Bausteine bereits reibungslos funktionieren.

\section{Die Balanced Scorecard (BSC)}

\subsection{Grundgedanke und Aufbau der BSC im Überblick}

Die Balanced Scorecard ist das in der Theorie als auch das in der Praxis am weitesten verbreitete Performance Measurement-System. Sie bietet einen konzeptionellen Handlungsrahmen, um den strategischen Führungsprozess im Unternehmen zu unterstuitzen. Dazu gliedern Kaplan und Norton das Steuerungssystem in vier Perspektiven (Finanzperspektive, Kundenperspektive, Interne Geschäftsprozessperspektive, Lern- und Entwicklungsperspektive) für die entsprechende Ziele, Kennzahlen und Maßnahmen zu entwickeln sind.

\subsubsection{Finanzperspektive}

Die finanzwirtschaftliche Perspektive soll darüber Auskunft geben, wie das Unternehmen die Interessen der Teilhaber befriedigen will. Finanzielle Kennzahlen sind trotz aller Kritik wesentlicher Bestandteil eines Steuerungssystems, da sie die wirtschaftlichen Konsequenzen früherer Aktionen aufzeigen [5]. Welche konkreten Messgrößen als finanzwirtschaftliche Endziele definiert werden, ist abhängig von der jeweiligen Geschäfts- strategie des Unternehmens, die wiederum stark vom Lebenszyklus der Geschäftseinheit beeinflusst wird.

Um in der Wachstumsphase die Entwicklung neuer Produkte und Dienstleistungen in neuen Märkten zu fördern, stehen entsprechende wachstumsorientierte Kennzahlen, wie z. B. Umsatzsteigerungsraten, im Vordergrund. In der Reifephase konzentriert sich das Management auf rentabilitätsorientierte Kennzahlen wie ROI oder wertorientierte Messgrößen wie EVA. In der letzten Phase des Lebenszyklusses wird versucht, die Potenziale der Geschäftseinheit zu liquidieren. Das Hauptziel in der Reifephase kann beispielsweise die Maximierung des Cashflow-Rückflusses sein.

Die finanzwirtschaftlichen Kennzahlen haben eine Richtgrößenfunktion für die Ziele der anderen Scorecardperspektiven.

\subsubsection{Kundenperspektive}

Die finanziellen Ziele, wie z. B. Umsatz- und Gewinnwachstum, sind nur erreichbar, wenn der Kunde dem Unternehmen mit dem Kauf von Produkten und Dienstleistungen Finanzmittel zufuihrt [6]. Erreichte Kundenziele sind somit Voraussetzung zur Erreichung der Finanzziele. Die Steuerungsgrößen der Kundenperspektive lassen sich in zwei Gruppen unterteilen:

In Ergebniskennzahlen, wie Marktanteile, Kundenzufriedenheit, Kundenakquisition und Kundentreue, die fuir fast alle Unternehmen anwendbar sind. Und in Leistungstreiberkennzahlen, die individuell ausdruicken, wodurch das Unternehmen einen definierten Zielwert einer Ergebniskennzahl erreichen will. D. h. welches Wertangebot das Unternehmen den Kunden bietet. Branchenuibergreifend sind drei Wertangebotsdimensionen zu unterscheiden [7]. Erstens die Produkt- und Dienstleistungseigenschaften, die in Zeit-, Qualitäts- und Preiskennzahlen ihren Ausdruck finden. Beispiele dafuir sind On-time-Delivery oder die Anzahl der Reklamationen.

Zweitens die Kundenbeziehungseigenschaften, die u. a. die Kompetenz, die Erreichbarkeit und Reaktionsgeschwindigkeit umfassen, welche beispielsweise mittels ausgebildeter Testkäufer ermittelt werden können.

Drittens das Image, das bei vielen Markenprodukten ausschlaggebend für den Kaufzuschlag ist und z. B. mit dem Preisaufschlag im Vergleich zu gleichwertigen Konkurrenzprodukten quantifiziert werden kann. Innerhalb der Kundenperspektive können so die finanziellen Ziele in steuerbare Kundenziele transformiert werden.

\subsubsection{Interne Geschäftsprozessperspektive}

Um die Ziele der Leistungstreiber in der Kundenperspektive hinsichtlich der Produkt- und Servicequalität ständig zu verbessern, müssen die dafür verantwortlichen Prozesse kontrolliert oder, falls nicht vorhanden, erst entwickelt werden. Die wertschöpfenden Prozesse im Unternehmen befinden sich in der Regel in der Entwicklung, der Produktion sowie im Vertrieb und Service. Die Innovationsfähigkeit als strategischer Erfolgsfaktor im Entwicklungsprozess kann beispielsweise durch den Prozentsatz des Umsatzes mit neuen Produkten oder durch die Anzahl der Produkte, welche die nächste Entwicklungsstufe erreicht haben, gemessen werden. 
Im Produktionsprozess wird die effiziente, beständige und pünktliche Herstellung und Lieferung existierender Produkte und Dienstleistungen an existierende Kunden herausgestellt. Mit Kennzahlen wie Durchlaufzeiten, Part-per-Million-Fehlerquoten oder Materialabfall kann die Leistung in produzierenden Unternehmen gemessen werden. Dienstleistungsunternehmen können sich z. B. der Wartezeiten oder Servicedauer zur Beurteilung der Leistung bedienen.

Der Kundendienst beinhaltet Garantie- und Wartungsarbeiten, die Bearbeitung von Fehlern und Reklamationen sowie die Abhandlung des Zahlungsverkehrs. Aus Häufigkeiten der oben beschriebenen Arbeiten lassen sich für diese Prozesse pragmatische Zielgrößen ableiten.

\subsubsection{Lern- und Entwicklungsperspektive}

Im Dienstleistungs- und Informationszeitalter werden motivierte Mitarbeiter sowie leistungsfähige Informationssysteme zu wettbewerbsentscheidenden Ressourcen, die helfen Geschäftsprozesse und Güter beständig weiterzuentwickeln. Neben der Mitarbeiterzufriedenheit können weitere Indikatoren zur Messung der Mitarbeitermotivation, wie z. B. die Anzahl der eingereichten bzw. umgesetzten Verbesserungsvorschläge oder die Mitarbeitertreue, hinzugezogen werden.
Wie eine Balanced Scorecard für ein Dienstleistungsunternehmen aussehen könnte, ist am Beispiel der Hamburger S-Bahn in der Abbildung 2 dargestellt.

\subsection{Das Managementsystem der Balanced Scorecard}

Das wahre Potenzial der BSC geht jedoch über die Verwendung als Kennzahlensystem hinaus und entfaltet sich erst bei der Nutzung als strategisches Managementsystem [8].

In den folgenden vier Managementprozessen wird erläutert, auf welche Weise Strategien operationalisiert werden können.

\subsubsection{Formulierung und Umsetzung von Vision und Strategie}

Die BSC sollte die Strategie einer Unternehmenseinheit durch eine Kette von Ursache-Wirkungsbeziehungen ausdrücken. Die Kausalbeziehung zwischen den verschiedenen Perspektiven, zwischen den strategischen Zielen und den entsprechenden Steuergrößen sowie zwischen den Ergebnis- und den Leistungstreiberkennzahlen des im Abschnitt 3.1 erläuterten Kennzahlensystems werden durch die sukzessive Aufstellung von Hypothesen erarbeitet und nach der Implementierung empirisch untersucht.

\begin{tabular}{l}
\hline \multicolumn{1}{c|}{ Finanzperspektive } \\
\hline stetige Umsatzsteigerung \\
(Umsatz im Jahr ${ }_{n}-$ Umsatz im Jahr $\left._{n-1}\right) /$ \\
Umsatz im Jahr $_{n-1}$ in Prozent
\end{tabular}

termingerechte Investition

Bruttoinvestitionen / Umsatz in Prozent

ertragsstarkes Wachstum

(Betriebsergebnis I +Abschreibungen)/

Umsatz in Prozent

angemessene Verzinsung

Betriebsergebnis I/

Capital Employed in Prozent

Interne Geschäftprozess Perspektive
pünktlichere Züge

Summe Züge mit maximaler Verspätung von

3 Min. an End.-Bf. / alle Züge in Prozent

sicherere Züge und Stationen

Anzahl Reisende / Anzahl Straftaten in

Tausend

sauberere Züge und Stationen

Fahrzeuge mit Qualitätswertzahl (QWZ)

Stationen mit Qualitätswertzahl (QWZ)

effizienter Einsatz Personal

Summe Zugkm / durchschnittliche

Beschäftigte in Tausend

\begin{tabular}{l} 
Kundenperspektive \\
\hline höherer Marktanteil \\
S-Bahn Pers.km / HVV Pers.km in Prozent \\
stärkere Auslastung \\
S-Bahn Pers.km / Sitzplatzkm in Prozent \\
zufriedenere Fahrgäste \\
Summe zufriedener Kunden / \\
Summe aller befragten Kunden in Prozent
\end{tabular}

besseres Image

Anzahl positive und ausgewogene Artikel /

gesamte S-Bahn-Artikel in Prozent

\section{Lern- und Entwicklungsperspektive \\ zufriedenere Mitarbeiter \\ Summe (sehr) zufriedener Mitarbeiter / \\ Summe aller befragten MA in Prozent \\ gesündere Mitarbeiter \\ 1 - (Summe Krankentage / \\ Summe Arbeitstage) in Prozent}

mehr Teilnehmer an Verbess. Maßnahmen Anzahl der MA die einen Verbesserungsvorschlag einreichen je $1.000 \mathrm{MA}$

zielorientiertes Handeln

durchgeführte Zielvereinbarungen $(Z V)+$

Mitarbeitergespräche (MAG) / geplante ZV +

MAG pro definierten Zeitraum in Prozent 
Im Entwicklungsprozess der BSC führt die Auseinandersetzung um diese Beziehungen zur Klärung und zum Konsens der Mitarbeiter hinsichtlich der Strategie.

\subsubsection{Kommunikation und Verbindung}

Um die Mitarbeiter und Führungskräfte auf eine Strategie auszurichten, müssen erstens gezielte Kommunikations- und Weiterbildungsprogramme durchgefuihrt werden, welche den Zweck und die Inhalte der vorher entwickelten BSC vermitteln. Zweitens müssen die Ziele der BSC bis hin zu den Zielvorgaben für Teams und Einzelpersonen heruntergebrochen werden. Drittens sollten diese persönlichen Ziele an ein Anreizsystem geknüpft werden. Eine Möglichkeit ist, bei der Vergabe von Boni und Prämien an Führungskräfte darauf zu achten, dass die Beurteilungsgrundlage aus allen Perspektiven Ziele enthält. Diese Ziele können unterschiedlich gewichtet werden, wobei jedoch ein bestimmtes Mindestniveau für alle Ziele erreicht werden sollte. Über die Qualität der gesetzten Ziele entscheidet der Vorgesetzte unter Zuhilfenahme von Vergleichsgrößen anderer Unternehmen [9].

\subsubsection{Planung und Vorgaben}

Neben der Steuerung der personellen Ressourcen sind auch die finanziellen und materiellen Ressourcen auf die Unternehmensstrategie auszurichten.

In drei Schritten erfolgt die Integration des strategischen Planungsprozesses [10]:

1. Stellen herausfordernder Ziele, um herkömmliche Denkweisen aufzubrechen.

2. Identifikation strategischer Initiativen, um die Soll-IstLücke der Leistung zu schließen. Aktionen werden nur durchgeführt, wenn sie maßgeblich und nachweisbar der Erreichung der gesetzten Ziele in der BSC dienlich sind.

3. Verbindung mit der Budgetierung, indem Investition nach ihrem strategischen Nutzwert priorisiert werden. Meilensteine, die in den definierten Kennzahlen Ausdruck finden, helfen, langfristige Projekte jährlich überprüfbar zu machen.

\subsubsection{Strategisches Lernen und Feedback}

Die Verbesserung der bestehenden Prozesse zur Erreichung festgesetzter strategischer Ziele bezeichnen Kaplan und Norton als ,Single-loop-Lernprozess'. Würde der Managementprozess an dieser Stelle enden, so würden Abweichungen von den festgelegten Plangrößen als Umsetzungsfehler interpretiert und Gegenmaßnahmen eingeleitet werden. Die Umfelder und damit die Basis, nach der die Strategie entwickelt wurde, ändern sich jedoch ständig. Deshalb benötigen Unternehmen die Fähigkeit zum ,Double-loop-Lernen' [11]. In diesem strategischen Lernprozess werden die Annahmen, auf denen die Strategie basiert, regelmäßig kritisch überprüft. Dies unterstuitzt die BSC, indem Korrelationen zwischen den Ursache-Wirkungsbeziehungen im Laufe der Zeit empirisch nachgewiesen werden und die Strategie gegebenenfalls entsprechend validiert und modifiziert wird. Mathematisch unbestechliche Zusammenhänge sind jedoch nicht zu erwarten. Mit Hilfe der BSC können Unternehmensplanspiele und Szenarioanalysen durchgeführt werden, die das Strategieverständnis des Managements erhöhen. Damit schließt sich der Kreis des Managementsystems der BSC und beginnt erneut bei der Formulierung und Umsetzung der Strategie.

\section{Das Quantum Performance Measurement Modell}

Das Quantum Performance Measurement Modell (QPMM) bietet ein systematisches Instrument zur Entwicklung, Implementierung und Nutzung ausbalancierter Steuerungsgrößen. Es gliedert sich in die vier Elemente Treiber, Befähiger, Prozess der Steuergrößenentwicklung sowie kontinuierliche Verbesserung (siehe Abbildung 3).

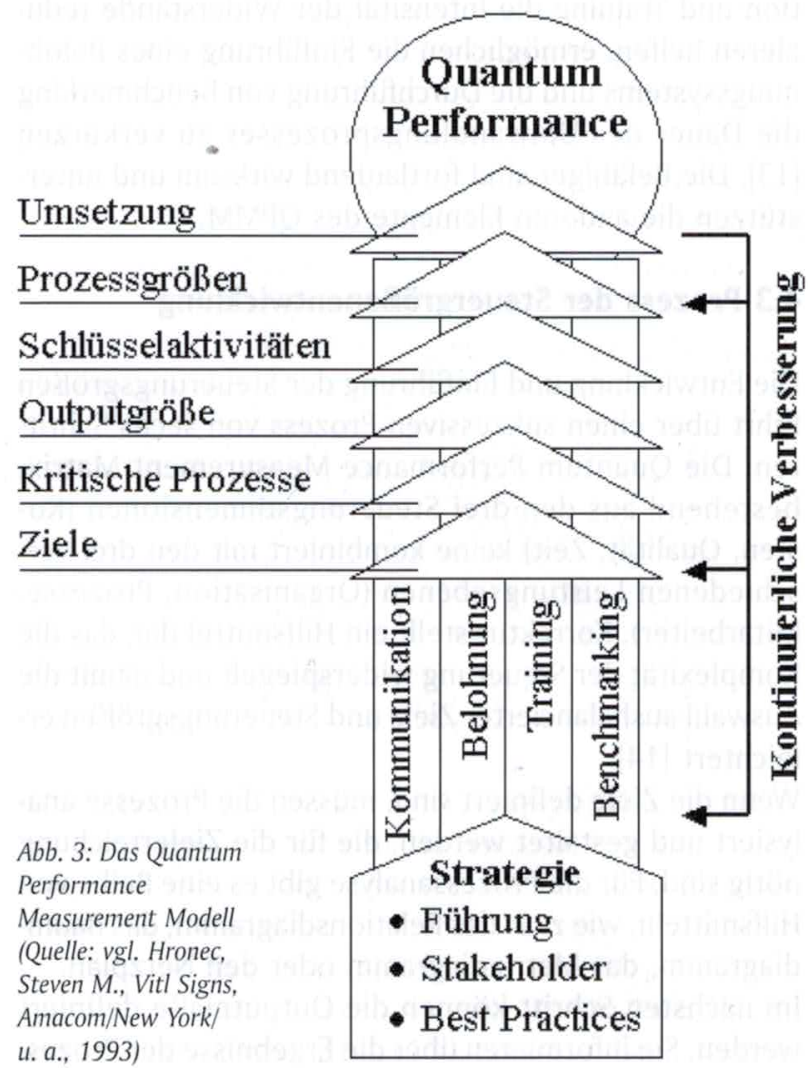

\subsection{Treiber}

Die Ausgangsbasis für die Entwicklung von ausbalancierten Steuerungsgrößen ist eine klare Strategie. Diese wird durch die drei Treiberquellen Stakeholder, Führung und Best Practice beeinflusst.

Die relevanten Stakeholder müssen identifiziert, ihre Wünsche und Erwartungen auf eine systematische Art und Weise analysiert und priorisiert werden. Dabei ist es wichtig, dass ein bilateraler Kommunikationsprozess stattfindet und auch die Stakeholder über den Stand des Unternehmens informiert werden [12]. Welchen Druck in der heutigen Zeit verschiedene Stakeholdergruppen auf ein Unternehmen ausüben können, zeigen die Fälle Shell gegen Greenpeace oder die Holocaust-Zwangsarbeiterentschädigung.

Die Aufgabe der Unternehmensfuihrung ist es, Unternehmensziele aus dem Interessensabgleich der Stakeholder 
heraus zu bestimmen. Das Management regelt die $\mathrm{Al}$ lokation der Ressourcen, die für die Zielerreichung nötig sind.

Um die dazu notwendigen Prozesse möglicht effizient und effektiv zu gestalten, sollte sich das Unternehmen bei der Zielsetzung an Best Practices orientieren. Dabei besteht der größte Nutzen in der Möglichkeit, den Mitarbeitern die Potenziale des Unternehmens aufzuzeigen und somit bisherige Denkmuster aufzubrechen.

\subsection{Befähiger}

Die Befähiger des QPMM unterstützen die Strategieumsetzung und die Ermittlung der Steuerungsgrößen. Der Wechsel eines Steuerungssystems in einer Organisation erzeugt Stress und Widerstände. Während Kommunikation und Training die Intensität der Widerstände reduzieren helfen, ermöglichen die Einführung eines Belohnungssystems und die Durchführung von Benchmarking die Dauer des Umwandlungsprozesses zu verkürzen [13]. Die Befähiger sind fortlaufend wirksam und unterstuitzen die anderen Elemente des QPMM.

\subsection{Prozess der Steuergrößenentwicklung}

Die Entwicklung und Einführung der Steuerungsgrößen führt über einen sukzessiven Prozess von sechs Schritten. Die Quantum Performance Measurement Matrix, bestehend aus den drei Steuerungsdimensionen (Kosten, Qualität, Zeit) keine kombiniert mit den drei verschiedenen Leistungsebenen (Organisation, Prozesse, Mitarbeiter), Korrektur stellt ein Hilfsmittel dar, das die Komplexität der Steuerung widerspiegelt und damit die Auswahl ausbalancierter Ziele und Steuerungsgrößen erleichtert [14].

Wenn die Ziele definiert sind, müssen die Prozesse analysiert und gestaltet werden, die für die Zielerreichung nötig sind. Für die Prozessanalyse gibt es eine Reihe von Hilfsmitteln, wie z. B. das Relationsdiagramm, das Baumdiagramm, das Matrixdiagramm oder den Netzplan.

Im nächsten Schritt können die Outputmaße definiert werden. Sie informieren über die Ergebnisse der Prozesse. Nachdem die im vorherigen Schritt analysierten Prozesse identifiziert wurden, müssen nun die Erwartungen der jeweiligen Prozessempfänger (interne und externe) ermittelt werden. Aus diesen ergeben sich Anhaltspunkte über die Art der Outputmaße [15]. Die Outputmaße können dann den einzelnen Zielen in der Quantum Performance Measurement Matrix zugeordnet werden. Im nächsten Schritt soll geklärt werden, wie die Outputgrößen durch die Steigerung der Effizienz und Effektivität der Schlüsselaktivitäten in den Prozessen verbessert werden können. Durch Process Mapping und Prozesskostenrechnung werden Potenziale in den Prozessen sichtbar. Es gibt zahlreiche Process Mapping-Techniken, wie z. B. Flowcharts, Work-flow-diagrams, Interfunktional Charts. Unterstützt werden diese Techniken durch eine Reihe von Problemfindungsmethoden, wie das Ursache-Wirkungs-Diagramm, Histogramme, Checklisten, Korrelationsdiagramme, Charts usw. Analysieren die Prozesseigner mit Hilfe der oben genannten Methoden ihre Arbeitsläufe, so können nichtwerttreibende
Aktivitäten identifiziert, eliminiert und die Prozesskomplexität ernorm reduziert werden [16].

Wie die Outputmaße bei den kritischen Prozessen, so informieren die Prozessmaße über die Schlüsselaktivitäten in den Prozessen. Mittels dieser Kenngrößen können Fortschritte in den Prozessen überwacht, Probleme frühzeitig erkannt und Schliusselaktivitäten verifiziert werden. Bei der Implementierung der Prozessmaße hilft wieder die Quantum Performance Measurement Matrix. Während auf der Prozessebene die Steuergrößen sowohl Output- als auch Prozessmaße sein können, bestehen auf der Mitarbeiterebene alle Steuergrößen aus Prozessmaßen [17].

Für eine erfolgreiche Implementierung ist die Akzeptanz und die Verlässlichkeit der Steuerungsgrößen entscheidend. Deshalb ist es erforderlich, die Personen die mit den Kennzahlen arbeiten sollen, in die Entwicklung mit einzubeziehen.

Ein zentrales Führungsteam soll mit Hilfe der Befähigerkomponenten die Mitarbeiter in die Ausgestaltung der Steuerungsgrößen integrieren. Weiterhin müssen geeignete Berichtsysteme entwickelt werden. Aus diesen wird ersichtlich, wann, wie oft und in welcher Art die Steuerungsgrößen erhoben werden sollen sowie wer für sie verantwortlich ist.

\subsection{Kontinuierliche Verbesserung}

Durch den regelmäßigen Abgleich zwischen der unternehmensinternen Leistungsfähigkeit und den unternehmensexternen Leistungsbedingungen wird eine kontinuierliche Weiterentwicklung ermöglicht. Hronec empfiehlt eine jährliche Überprïung der Prozesse, Ziele und Strategien mittels des QPMM, um eine kontinuierliche Verbesserung zu erreichen.

\section{Das European Foundation for Quality Management-Excellence Modell (EFQM-Modell)}

\subsection{Grundlagen}

Während die beiden zuvor dargestellten Performance Measurement-Systeme in ihrer Entwicklungsgeschichte als Controllinginstrumente Verbreitung gefunden haben, liegt der Ursprung des EFQM-Modells im Total Quality Management (TQM). Die Ziele und die prinzipiellen Überlegungen, die dem EFQM-Modell zu Grunde liegen, decken sich jedoch erstaunlich gut mit dem bereits erläuterten Performance Measurement-Verständnis.

Um die Wettbewerbsfähigkeit europäischer Unternehmen durch die Verbreitung des TQM-Ansatzes zu fördern, wurde erstmals 1992 der European Quality Award (EQA) verliehen. Diesem liegt das EFQM-Modell als strategisches Rahmenmodell zu Grunde, das als Diagnose- und Bewertungsinstrument dabei hilft, die Organisation zu führen und Verbesserungsmöglichkeiten zu identifizieren [18]. Wie das EFQM-Modell aufgebaut ist und wie es dazu beiträgt, durch eine systematische Messung die Unternehmensleistung stetig zu verbessern, ist Inhalt der folgenden Abschnitte. 


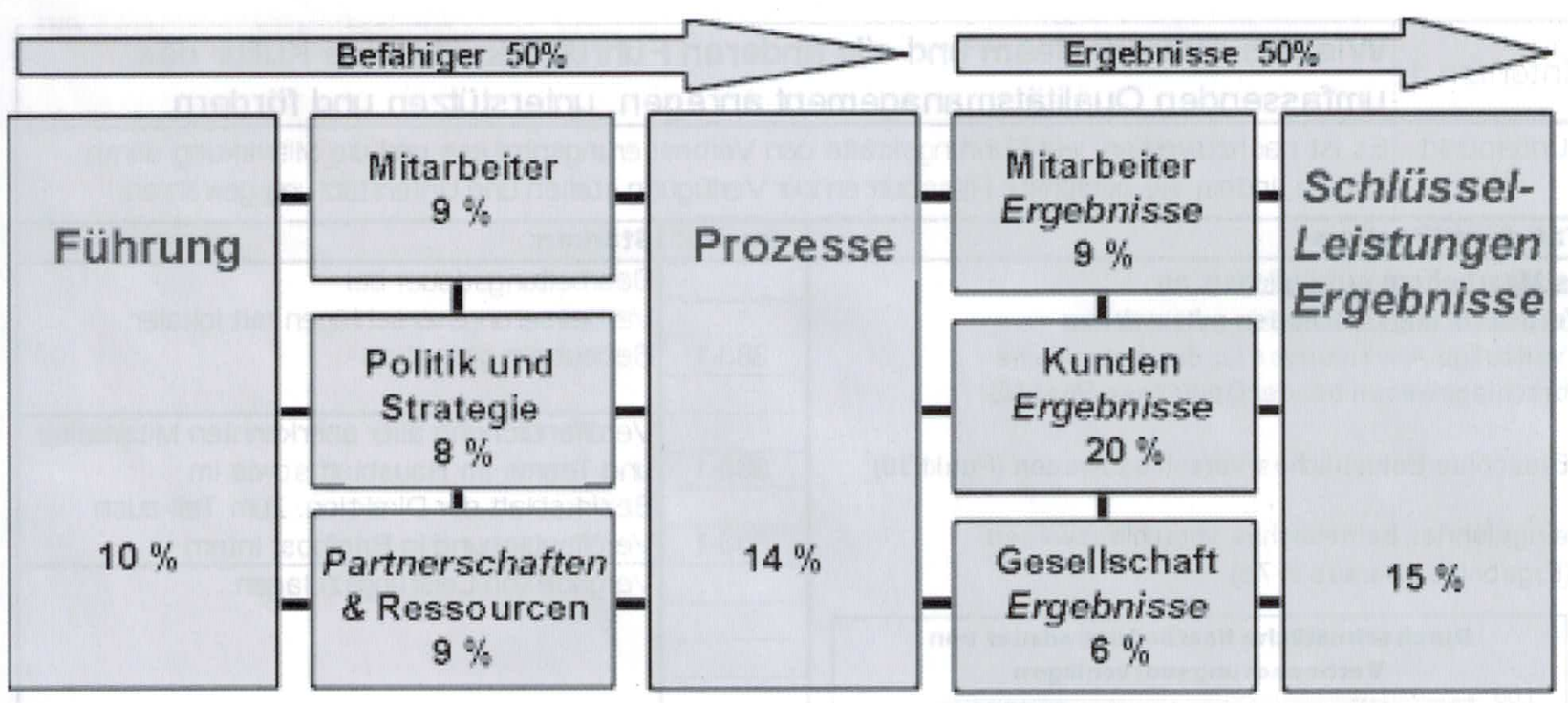

\section{Innovation und Lernen}

EFQN

Abb. 4: Das EFQM-Modell für das Jahr 2000 (Quelle: o.V.: Homepage der EFQM in Deutschland, http://www.deutsche-efqm.de/efqm/modell2000-1.html, 24.05.2000)

\subsection{Elemente des EFQM-Modells}

Dem EFQM-Modell liegt die Erkenntnis bzw. das Postulat zu Grunde, dass die Kunden- und Mitarbeiterzufriedenheit sowie eine allgemein positive Wirkung auf die Gesellschaft durch eine entsprechende Unternehmensfuihrung erzielt werden, welche die Politik und Strategie, die Mitarbeiterfuihrung sowie das Management der Ressourcen und Prozesse vorantreibt, was letztlich zu exzellenten Geschäftsergebnissen fuihrt [19].

Das EFQM-Modell besteht aus zwei wesentlichen Teilen, aus den fünf Befähiger-Kriterien und vier Ergebnis-Kriterien (siehe Abbildung 4). Die 9 Kriterien konkretisieren sich in ca. 33 Unterkriterien und 167 Ansatzpunkten, die in einem Fragenkatalog aufgelistet sind.

Weil mittels des EFQM-Fragenkatalogs die Managementprozesse und die Ergebnisse furr die verschiedenen Anspruchsgruppen, nicht aber die Gestaltung der unternehmensspezifischen Geschäftsprozesse bewertet werden, ist dieses Modell für Unternehmen aller Branchen und Größen anwendbar.

Die Befähiger-Kriterien befassen sich damit, wie das Unternehmen bei jedem Unterpunkt eines Kriteriums vorgeht, während die Ergebnis-Kriterien sich darauf beziehen, was das Unternehmen erreicht hat [20].

\subsection{Ablauf der Selbstbewertung}

Der Selbstbewertungsprozess folgt einem Regelkreislauf. Dieser ermöglicht es dem Unternehmen, seine Stärken und Schwächen klar zu erkennen, Ziele abzuleiten und Verbesserungsmaßnahmen umzusetzen.

Nachdem die Führungskräfte mit dem EFQM-Modell vertraut gemacht, die Assessoren ausgebildet und für die Kriterien Selbstbewertungsteams gebildet wurden, wird bei der erstmaligen Durchführung des Selbstbewertungsprozesses mit der Erhebung des Ist-Zustandes begonnen.

\subsubsection{Selbstbewertung im engeren Sinne}

Um die Unternehmensqualität messbar zu machen, werden Punkte für jedes Unterkriterium verteilt. Die Summe aller möglichen Punkte beträgt 1000 . Während die Unterkriterien innerhalb des Kriteriums meistens gleichgewichtet sind, so sind die Kriterien selbst unterschiedlich gewichtet. Um die Ergebnisse zwischen den Unternehmen vergleichbar zu machen, wird jedes Unterkriterium anhand einer definierten Bewertungsmatrix bepunktet. Während dabei die Befähiger-Kriterien nach dem Vorgehen, der Umsetzung und der Überprüfbarkeit der eingeführten Managementprozesse beurteilt werden, bewerten die Assessoren die Ergebnis-Kriterien u. a. nach Best Practise sowie den zuvor gesetzten Zielen.

Mittels des Fragenkataloges und der Bewertungsmatrix kann für jede Unternehmenseinheit eine separate Selbstbewertung durchgefuihrt werden. Oberstes Prinzip ist, dass alle Angaben durch Zahlen, Daten oder Fakten belegbar sein müssen. Um die Datensammlung zu vereinfachen, können standardisierte Formblätter genutzt werden, um den Leistungsstand $\mathrm{zu}$ beschreiben und Nachweise aufzulisten. In der Abbildung 5 ist am Beispiel der Deutschen Post ein Ausschnitt eines solchen Formblattes für das Unterkriterium 1b dargestellt. Die Ansatzpunkte (in der Abbildung unterstrichen dargestellt) der jeweiligen Unterkriterien helfen dabei, die Bewertungserwartungen zu konkretisieren. Auf den besagten Formblättern lassen sich auch Stärken und Verbesserungsbereiche für die jeweiligen Kriterien identifizieren. Diese Informationen bilden die Basis für die anschließende Auswertungsphase.

\subsubsection{Ableitung von Verbesserungsmaßnahmen}

Der Fragenkatalog der EFQM ist ein mehrdimensionales Diagnoseinstrument, mit dem verschiedene TQMSteuerungsansätze abgefragt und mittels der Ansatzpunkte konkrete Gestaltungsempfehlungen für deren Umsetzung abgeleitet werden können. 


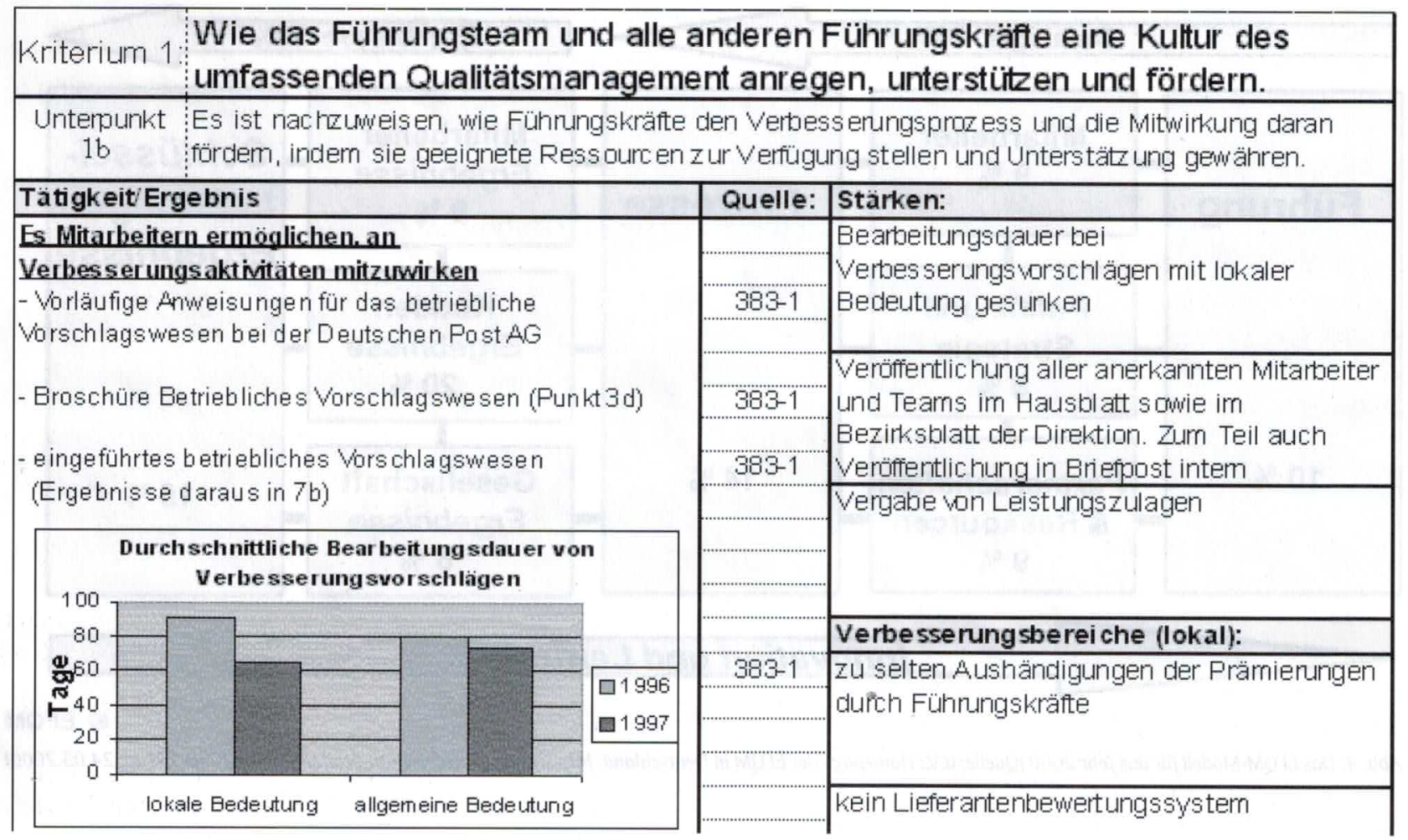

Abb. 5: Ausschnitt aus Muster-Kursbuchblatt der Niederlassungen der Deutschen Post

Das EFQM-Modell eignet sich aufgrund der einheitlichen Bewertungsgrundlage und des quantifizierbaren Erfolgs im besonderen Maße als Benchmarkingbasis. Da sich die EQA-Gewinner verpflichten, ihre Umsetzungserfahrungen weiterzugeben, setzen sie somit Maßstäbe und geben gleichzeitig Einblicke in die Gestaltung von Best Practice-Ansätzen. Die EFQM unterstuitzt europäische Unternehmen darüber hinaus durch die Publikation von Fallstudien, die den Unternehmen Verbesserungsanregungen geben können. Die relativierende Eigenschaft des Benchmarkings erleichtert die Konsensfindung im Team bezüglich der Beurteilung des Geleisteten und der noch bevorstehenden Herausforderungen. Es liefert objektive Daten statt Meinungen und unterstuitzt somit die Idee eines Management by Facts. Aus dem Vergleich des Erreichten und dem des Möglichen ergeben sich konkret für jedes einzelne Unterkriterium Hinweise auf realisierbare Verbesserungen.

\subsubsection{Zielfindung}

Aus den Ergebnissen der Selbstbewertung und des Benchmarkings lassen sich zahlreiche operative Verbesserungsmaßnahmen ableiten. Damit diese nicht unstrukturiert und zu Lasten anderer Bereiche realisiert werden, müssen sie mit der Strategie verknüpft werden. Dabei können eindimensionale Zielfindungsmethoden zu Suboptimierungen fuihren. Mehrdimensionale Konzepte (Policy DeploymentKonzepte), wie z. B. das Hoshin Planning oder das Goal

\begin{tabular}{|c|c|c|c|}
\hline Charakteristika & $\begin{array}{l}\text { BSC } \\
\text { Autor: Kaplan und Norton } \\
\text { Buchveröffentlichung: } 1996 \\
\end{array}$ & $\begin{array}{l}\text { EFQM-Modell } \\
\text { Autor: EFQM } \\
\text { Modellveröffentlichung: } 1991 \\
\end{array}$ & $\begin{array}{l}\text { QPMM } \\
\text { Autor: Steven Hronce } \\
\text { Buchverüffentlichung: } 1993\end{array}$ \\
\hline $\begin{array}{l}\text { Strategie- } \\
\text { orientierung }\end{array}$ & $\begin{array}{l}\text { Durch Erarbeitung und Uberprüfung der } \\
\text { Ursache-Wirkungsbeziehungen. }\end{array}$ & $\begin{array}{l}\text { Durch Beantwortung des Kriteriums } \\
\text {,Politik \& Strategie'. }\end{array}$ & Durch die ,Treiber' des QPMM. \\
\hline $\begin{array}{l}\text { Berücksichti- } \\
\text { gung der } \\
\text { relevanten } \\
\text { Stakeholder }\end{array}$ & $\begin{array}{l}\text { Durch perspektivische Betrachtungs- } \\
\text { weise (Teilhaber, Kunden, Mitarbeiter). } \\
\text { Erweiterung auf weitere } \\
\text { Anspruchsgruppen möglich. }\end{array}$ & $\begin{array}{l}\text { Z.B. durch die vier Ergebniskriterien } \\
\text { Kunden-, Mitarbeiter-, Gesellschafts- } \\
\text { und Schlüsselergebnisse. }\end{array}$ & $\begin{array}{l}\text { Stakeholder werden bei Strategieent- } \\
\text { wicklung und Kennzahlendefinition } \\
\text { berücksichtigt. }\end{array}$ \\
\hline $\begin{array}{l}\text { Orientierung } \\
\text { an kritischen } \\
\text { Prozessen }\end{array}$ & $\begin{array}{l}\text { Interne Geschäftsprozessperspektive. } \\
\text { Kritische Prozesse an } \\
\text { Wertschöpfungskette orientiert. }\end{array}$ & $\begin{array}{l}\text { Befähigerkriterien hinterfragen } \\
\text { Managementprozesse. Kriterium ,Pro- } \\
\text { zesse' analysiert Prozessmanagement. }\end{array}$ & $\begin{array}{l}\text { Konzeptioneller Bestandteil des QPMM. } \\
\text {,Prozess' als Objektebene in QPM- } \\
\text { Matrix berücksichtig. }\end{array}$ \\
\hline $\begin{array}{l}\text { Festlegung } \\
\text { Ebenen- } \\
\text { spezifische } \\
\text { Messgrößen }\end{array}$ & $\begin{array}{l}\text { Ausbalancierte Kennzahlen } \\
\text { heruntergebrochen mittels Ursache- } \\
\text { Wirkungsketten. Ableitung über die vier } \\
\text { Perspektiven in fester Reihenfolge. }\end{array}$ & $\begin{array}{l}\text { Gesamtpunktzahl für jede } \\
\text { Unternehmenseinheit mit eigener } \\
\text { Strategie ermittelbar. Ergibt sich aus } \\
\text { Summe der Punkte der Unterkriterien. }\end{array}$ & $\begin{array}{l}\text { Ergeben sich aus Analyse der kritischen } \\
\text { Prozesse und Schlüsselaktivitäten. Mit } \\
\text { QPM-Matrix werden Leistungsebenen } \\
\text { ausbalancierte Kennzahlen zugeordnet. }\end{array}$ \\
\hline Zielbildung & $\begin{array}{l}\text { In Zielfindungsgesprächen sollen } \\
\text { hochgesteckte Ziele gesetzt werden. }\end{array}$ & $\begin{array}{l}\text { Sowohl durch } \mathrm{MbO} \text { als auch durch } \\
\text { Quality Function Deployment. }\end{array}$ & $\begin{array}{l}\text { Z.B. durch Benchmarking sowie Target- } \\
\text { costing. }\end{array}$ \\
\hline $\begin{array}{l}\text { Maßnahmen- } \\
\text { ableitung }\end{array}$ & $\begin{array}{l}\text { Aus operationalisierten Kennzahlen und } \\
\text { deren Zielausprägungen. }\end{array}$ & $\begin{array}{l}\text { Aus Stärken-Schwächen-Analyse, aus } \\
\text {,Best Practice' und ,Anhaltspunkten'. }\end{array}$ & $\begin{array}{l}\text { Aus Analyse der kritischen Prozesse und } \\
\text { der Schlüsselaktivitäten. }\end{array}$ \\
\hline Messung & Durch das Kennzahlensystem der BSC. & $\begin{array}{l}\text { Mittels Fragenkatalog und } \\
\text { Bewertungsmatrix. }\end{array}$ & $\begin{array}{l}\text { Mit Kennzahlen der Quantum } \\
\text { Performance Measurement-Matrix. }\end{array}$ \\
\hline $\begin{array}{l}\text { Kontinuierliche } \\
\text { Verbesserung }\end{array}$ & $\begin{array}{l}\text { Durch „Single-loop- und Double-loop- } \\
\text { Lernenprozess" }\end{array}$ & $\begin{array}{l}\text { Durch regelmäßiges Selbstbewertungs- } \\
\text { zyklen. }\end{array}$ & $\begin{array}{l}\text { Regelmäßige Überprüfung der Strategie, } \\
\text { der Ziele und der Prozessgrößen. }\end{array}$ \\
\hline Visualisienung & Durch vier Perspektiven. & Durch EFQM-Modell. & Durch QPM-Modell. \\
\hline Anreizsysteme & $\begin{array}{l}\text { Auch finanzielle Anreize für } \\
\text { Führungskräfte z.B. in Form von Boni. }\end{array}$ & $\begin{array}{l}\text { Betonung nichtfinanzieller Anreize, wie } \\
\text { z.B. Belobigungen. }\end{array}$ & $\begin{array}{l}\text { Hervorhebung der Bedeutung der nicht- } \\
\text { finanziellen Anreize. }\end{array}$ \\
\hline
\end{tabular}


Deployment, versuchen eine enge Verknuipfung des vertikalen Herunterbrechens der Ziele über die Hierarchieebenen hinweg mit der horizontalen Zielableitung entlang der Prozesskette zu erreichen [21]. Im Unterschied zum $\mathrm{MbO}$ wird beim Goal Deployment besonders darauf geachtet, dass erst geeignete Strategien und Maßnahmen zur Zielerreichung auf der betreffenden Ebene entwickelt werden, bevor die Ziele endgültig festgeschrieben und Teilziele weiter heruntergebrochen werden.

Bevor die gesetzten Ziele und die davon abgeleiteten Maßnahmen umgesetzt werden können, müssen die entsprechenden Initiativen geplant werden. Dazu zählen die Zuweisung von Verantwortlichkeiten sowie die Planung der zeitlichen und finanziellen Ressourcen.

\subsubsection{Maßnahmenumsetzung}

Nach der Planung erfolgt die Umsetzung der wichtigsten Verbesserungsmaßnahmen, die bei der Bewertung identifiziert und nach strategischen Gesichtspunkten priorisiert worden sind. Die größte Herausforderung bei der Umsetzung ist die langfristige Integrierung der entwickelten Maßnahmen in das operative Geschäft. Das bedeutet, dass sich die Organisationsstrukturen und die Prozesse flexibel den neuen Aufgaben anpassen müssen. Gleichermaßen muss der Führungsstil die Bereitschaft zu Veränderung fördern. Dazu zählt die Übertragung von Verantwortung auf die operativen Ebenen genauso wie die Etablierung von qualitätsorientierten Anreizsystemen.

Der Selbstbewertungsprozess kann regelmäßig wiederholt werden. Aufgrund des hohen Aufwandes empfiehlt sich ein jährlicher Bewertungsrhythmus.

\section{Zusammenfassung und Ausblick}

Im Ergebnis der Betrachtung der einzelnen Performance Measurement-Systeme lässt sich festhalten, dass die Modelle nicht alternativ zu sehen sind. So können sich die BSC und das EFQM-Modell in ihrer Entwicklung gegenseitig positiv beeinflussen.

Mit dem EFQM-Modell können dank der Stärken- und Schwächenanalyse konkrete Verbesserungsmaßnahmen identifiziert werden. Damit werden gleichzeitig Inhalte und Strukturen fuir die Entwicklung und Ausgestaltung des BSC sichtbar. Die BSC liefert im Gegenzug für diese Maßnahmen individuelle Meßgrößen, mit denen kontinuierlich der Fortschritt sowohl operativ als auch strategisch gesteuert werden kann. Auf diese Weise vereinfacht und beschleunigt die BSC den Datenerhebungsprozess der anschließenden EFQM-Selbstbewertung durch die Bereitstellung eines quantifizierten Bewertungsinputs.

Auch einzelne Elemente des QPMM, wie z.B. die QPMMatrix, die einen strukturierten Ansatz zur Entwicklung mehrdimensionaler Steuerungsgrößen bietet, können genutzt werden, um ein den individuellen Anforderungen entsprechendes Steuerungsinstrument zu entwerfen.

Nur ein Steuerungsinstrument, mit dessen Hilfe die individuelle Strategie in allen erfolgskritischen Bereichen anspruchgruppengerecht quantifiziert werden kann und welches dazu beiträgt, die Leistung der Prozesse kontinuierlich zu verbessern, vermag einer Unternehmung auch zukiinftig die Handlungsfähigkeit zu sichern.
Deshalb wird der Einsatz eines Performance Measurement-Systems für Unternehmen, die in dynamischen Märkten agieren, zur erfolgsnotwendigen Navigationshilfe werden [22].

\section{Anmerkungen}

[1] vgl. Fritsch, Michael: Performance Management - der Mensch als Erfolgsfaktor, in: Marktforschung \& Management, 39. Jg., 1994, Nr. 2, S. 48-54.

[2] vgl. Sure, Matthias/Haselgruber, Boris: Balanced Scorecard - ein strategisches Instrument zur Unternehmenssteuerung, in: Zeitschrift für Unternehmensentwicklung und Industrial Engineering, 48. Jg., 1/1999, S. 4.

[3] Gleich, Ronald: Performance Measurement, in: Die Betriebswirtschaft, 1997, S.115 (A).

[4] Klingebiel, Norbert, Performance Measurement: Grundlagen - Ansätze - Fallstudien, Wiesbaden, 1999, S. 3 (A)

[5] vgl. Kaplân, Robert S./Norton, David P.: Balanced Scorecard, Stuttgart, 1997 (A), S. 24

[6] vgl. Krahe, Andreas: Balanced Scorecard - Baustein zu einem prozeßorientierten Controlling?, in: controller magazin, 2/1999, S.117

[7] vgl. Kaplan, Robert S./Norton, David P.: Balanced Scorecard, Stuttgart, 1997 (A), S. 71 ff.

[8] vgl. ebd., S. 10

[9] vgl. ebd., S. $209 \mathrm{ff}$.

[10] vgl. edb., S. 216

[11] vgl. ebd., S. 243

[12] vgl. Hronec, Steven M.: Vitl Signs, Amacom/New York/ u. a., 1993, S. 61

[13] vgl. ebd., S. 79

[14] vgl. Gleich, Ronald: Performance Measurement, in: controlling, Sept./Okt. 1997, S. 365 (B)

[15] vgl. Hronec, Steven M., Vitl Signs, Amacom/New York/ u. a., 1993 , S. $128 \mathrm{ff}$.

[16] vgl. ebd., S. $152 \mathrm{ff}$.

[17] vgl. ebd., S. $187 \mathrm{f}$.

[18] vgl. European Foundation for Quality Management (Hrsg.): Selbstbewertung - Richtlinien für Unternehmen 1998, 6. Jg., Tilburg, 1996, S. 33

[19] vgl. ebd. S. 9

[20] vgl. European Foundation for Quality Management (Hrsg.), a.a.O., S. $10 \mathrm{f}$.

[21] vgl. Zink/Klaus, J.: TQM als integratives Managementkonzept, München/Wien, 1995, S. $279 \mathrm{ff}$.

[22] vgl. Gleich, Ronald, a.a.O., S. 116 (A)

\section{Autor}

Dipl.-Betriebswirt (FH) Christian Klatte

Junior Berater bei Dr. Göhring \& Partner

Unternehmensberatung AG

Schützenhofstr. 3, 65183 Wiesbaden

Tel. (06 11) 398 95-0

E-Mail:klatte@g-und-p.de 